\title{
Estratificação do risco cardiovascular em cadeirantes jogadores de basquetebol
}

\author{
Kelen Cristina Estavanate de Castro \\ Ana Clara Garcia Guimarães \\ Guilherme Junio Silva \\ Marconi Guarienti \\ Maria Georgina Marques Tonello \\ Olímpio Pereira de Melo Neto \\ Karine Cristine de Almeida \\ Daniel dos Santos
}

\begin{abstract}
Resumo: Objetivou-se estratificar fatores de risco para doenças cardiovasculares (DCV) em dez anos em jogadores de basquetebol em cadeiras de rodas. O percentual de risco cardiovascular foi estratificado pelos escores de Framingham (ERF) e de Risco Global (ERG). Dos treze jogadores avaliados, $38,46 \%$ apresentava sobrepeso e obesidade e $77 \%$, alterações na porcentagem de gordura corporal e na circunferência abdominal. O ERF identificou 15,38\% dos jogadores com risco intermediário para desenvolvimento de DCV e pelo ERG, $15,4 \%$ dos homens apresentava risco intermediário, $7,7 \%$ alto risco por diabetes e 7,7\% muito alto risco por doenças ateroscleróticas. Os resultados de composição corporal demonstram uma predisposição para desenvolvimento de DCV, sendo que o ERG identificou indivíduos com alto risco e muito alto risco. Conclui-se que a identificação dos fatores de risco é útil para que ocorra uma maior prevenção das DCV e aconselhamento adequado sobre adoção de hábitos de vida saudáveis por esses indivíduos.

Palavras chave: Defíciência física; Doenças cardiovasculares; Fatores de risco.
\end{abstract}

\section{Cardiovascular risk stratification in wheelchair basketball players}

Abstract: The objective of this study was to stratify risk factors for cardiovascular diseases (CVD) in ten years in wheelchair basketball players. The percentage of cardiovascular risk was stratified by the Framingham Score (FS) and Global Risk Score (GRS). Of the thirteen players evaluated, $38.46 \%$ were overweight and obese and $77 \%$, presented changes in body fat percentage and waist circumference. The GRS identified $15.38 \%$ of the players with intermediate risk for developing CVD and according to GRS, $15.4 \%$ of men presented intermediate risk, 7.7\% high risk for diabetes and $7.7 \%$ very high risk for atherosclerotic diseases. The results of body composition demonstrate a predisposition for the development of CVD and the ERG identified individuals with high risk and very high risk. It is concluded that the identification of risk factors is useful for a greater prevention of CVD and appropriate advice on the adoption of healthy lifestyle habits by these individuals.

Key words: Physical disability; Cardiovascular diseases; Risk factors.

\section{Introdução}

As doenças cardiovasculares (DCV) são a principal causa de morte no mundo, sendo que, em 2015, 17,7 milhões de pessoas foram vítimas de doenças do coração ou dos vasos sanguíneos, representando $31 \%$ de todas as mortes em nível global (Gama et al., 2017; World Health Organization [WHO], 2017). No Brasil, as DCV são responsáveis 
pela morte de pelo menos $20 \%$ da população com mais 30 anos de idade, principalmente nas regiões sul e sudeste do país (Mansur \& Favarato, 2016).

Os principais fatores de risco comportamentais para as DCV são dietas inadequadas, sedentarismo, uso de tabaco e uso nocivo do álcool. Tais fatores podem se manifestar em indivíduos por meio de pressão arterial elevada, glicemia alta, hiperlipidemia, sobrepeso e obesidade, indicando um maior risco de desenvolvimento de ataques cardíacos, acidentes vasculares cerebrais, insuficiência cardíaca e outras complicações (Filho, Salvetti, Mello, Silva \& Filho, 2006; WHO, 2017). Mudanças no estilo de vida e controle dos fatores de risco, têm se mostrado eficazes para reduzir o risco de DCV (Freire et al., 2017; WHO, 2017).

Levando-se em conta que as DCV são doenças não transmissíveis, sua prevenção e tratamento vai ao encontro do terceiro Objetivo de Desenvolvimento Sustentável (ODS) da Organização das Nações Unidas (ONU) no item 3.4, que propõe a redução em um terço da mortalidade prematura por essas doenças até 2030 (Organização das Nações Unidas [ONU], 2015).

Nesse sentido, o diagnóstico precoce por meio da identificação de um ou mais fatores de risco, torna-se fundamental para prevenção da ocorrência das DCV. Principalmente em indivíduos que apresentem uma maior predisposição à doença, como deficientes físicos com lesão na coluna vertebral, uma vez que apresentam uma maior deposição de gordura na região abdominal (Santos \& Guimarães, 2002; Donatto et al. 2008; Quintana \& Neiva 2008; Eskici \& Ersoy, 2016). Estudos indicam que mesmo entre atletas profissionais, deficientes físicos, são identificadas condições como diabetes mellitus (DM), sobrepeso e obesidade, o que predispõe estes indivíduos à ocorrência das DCV (Platt, 2001).

Para a identificação dos fatores de risco que contribuem para as DCV, a Sociedade Brasileira de Cardiologia (SBC) recomenda, na Atualização da Diretriz Brasileira de Dislipidemias e Prevenção da Aterosclerose (2017), o uso do Escore de Risco Global (ERG) que estima o risco de infarto do miocárdio, AVC, ou insuficiência cardíaca, fatais ou não fatais, ou insuficiência vascular periférica em 10 anos. Este escore foi desenvolvido a partir do Framingham Heart Study, um escore que estratifica o risco cardiovascular em 10 anos pela probabilidade de um evento coronariano em indivíduos sem diagnóstico anterior de aterosclerose (Faludi et al., 2017; Gama et al., 2017; Cezar-Vaz et al., 2018).

A estratificação do risco cardiovascular compreende o somatório dos fatores de risco com a finalidade de indicar o grau da ameaça para desenvolvimento de doenças coronarianas (Candido, Faria, Silva \& Dantas, 2016). O escore de Framingham (ERF) atribui pontuações para a idade, colesterol total, HDL colesterol, pressão arterial sistólica, tratamento para hipertensão e fumo. A atribuição de pontos e percentual de risco é diferenciada para homens e mulheres. Já o ERG, utiliza os mesmos parâmetros acima e deve ser utilizado na avaliação inicial de indivíduos não enquadrados em condições de alto risco como a presença de DM e doenças ateroscleróticas (DA) (Faludi et al., 2017; Malachias et al., 2016). Embora sejam escores simples, de baixo custo e seguros para a estratificação do risco cardiovascular a SBC recomenda o uso de mais de um escore para basear as decisões terapêuticas, devido à ausência de dados brasileiros para uma avaliação mais específica do risco cardiovascular (Malachias et al., 2016). 
Vários estudos foram conduzidos com a utilização destes escores para avaliar o risco de populações de desenvolverem doenças cardiovasculares (Oliveira et al., 2007; Gama et al., 2017; Jahangiry, Farhangi \& Rezaei, 2017; Cezar-Vaz et al., 2018). No entanto, poucos estudos têm sido conduzidos com objetivo de avaliar a prevalência de fatores de risco coronarianos em deficientes físicos (Filho et al., 2006).

Diante da importância das DCV como um problema de saúde pública global, este artigo teve como objetivo identificar e estratificar fatores de risco para eventos cardiovasculares em 10 anos em jogadores de basquetebol em cadeira de rodas.

\section{Método}

Esta pesquisa fez parte de um estudo maior denominado "Contribuição da intervenção nutricional para a redução do risco de doenças cardiovasculares e melhora da qualidade de vida de jogadores de basquetebol em cadeira de rodas", aprovada pelo Comitê de Ética em Pesquisa do Centro Universitário de Patos de Minas (UNIPAM) em fevereiro de 2018, sob parecer $\mathrm{n}^{\mathrm{o}} 2.494 .455$.

Trata-se de um estudo transversal, quantitativo e descritivo, realizado em março de 2018, com jogadores de basquetebol em cadeira de rodas de uma equipe do Alto Paranaíba, que participaram como voluntários da pesquisa. Todos os jogadores que se reúnem periodicamente para treinamento no ginásio poliesportivo do UNIPAM foram convidados a participar da pesquisa. Foram incluídos os jogadores praticantes da atividade há mais de trinta dias e que aceitaram participar da pesquisa mediante assinatura de um termo de consentimento livre e esclarecido autorizando a avaliação e o uso de seus dados. Foram excluídos os jogadores que estavam em uso de medicação para emagrecimento, controle do perfil lipídico ou controle da glicemia, e que não estavam presentes no dia da avaliação.

A coleta de dados foi realizada em março de 2018, nos laboratórios de avaliação nutricional e de análises clínicas do UNIPAM. Os jogadores foram avaliados no período da manhã após repouso de 12 horas, desde a última sessão de treinamento.

Os métodos de avaliação incluíram aplicação de questionário sócio demográfico, no qual foram registradas informações como dados pessoais, histórico médico, uso de medicamentos e bebidas alcoólicas, dados da composição corporal e bioquímicos com o objetivo de caracterizar a amostra além de elucidar fatores de risco cardiovascular como fumo e histórico familiar.

Para realização da avaliação hemodinâmica os jogadores foram orientados a permanecerem em repouso por dez minutos sentados na cadeira de rodas. A pressão arterial (PA) foi obtida por método de ausculta com a utilização de estetoscópio, modelo Tycos ${ }^{\circledR}$ e esfigmomanômetro coluna de mercúrio modelo Missouri ${ }^{\circledR}$, aferidos pelo INMETRO. A medida foi dada em milímetros de mercúrio $(\mathrm{mmHg})$ observando-se as recomendações para aferição da PA segundo a $7^{\mathrm{a}}$ Diretriz Brasileira de Hipertensão Arterial da Sociedade Brasileira de Cardiologia. Para determinação da pressão arterial sistólica (PAS) foi considerada a fase I de Korotkoff, e a fase V para a pressão arterial diastólica (PAD). Os valores de PA encontrados foram comparados com as referências para adultos da mesma diretriz (Malachias et al., 2016). 
Para a avaliação antropométrica foram aferidas as medidas de estatura e massa corporal de cada jogador para o cálculo do Índice de Massa Corporal (IMC), definido como o peso do indivíduo em quilogramas dividido pelo quadrado da altura em metros. Foram considerados com excesso de peso e obesos os participantes que apresentaram valores de IMC de 25,0 - 29,9 e > 30,0 Kg/m2, respectivamente. Para a avaliação do risco de complicações metabólicas, foi aferida a medida da circunferência da cintura (CC) e os valores comparados com as recomendações (risco aumentado $\geq 94$ para homens e $\geq$ 80 para mulheres e risco substancialmente aumentado $\geq 102$ para homens e $\geq 88$ para mulheres). Os dados de estatura e CC foram utilizados para calcular índice de obesidade central (ICO), considerado por Rossi (2017) como preditor de risco cardiovascular para homens e mulheres. Os valores encontrados foram comparados com as referências para homens: de 0,51 a 0,58. Foi utilizado o cálculo de Bulbulian, Johnson, Gruber e Darabos (1987) que utiliza o combinado de medidas de diâmetro, dobras cutâneas e circunferências corporais para mensurar a densidade corporal (DC) e calcular o percentual de gordura corporal (\%GC) de cada jogador (Rossi, 2017).

Para a obtenção da amostra sanguínea os jogadores foram submetidos a 12 horas de jejum, com restrição no consumo de bebidas alcoólicas pelo período de 72 horas e repouso de 30 minutos antes da coleta no período da manhã. A coleta foi realizada por profissional farmacêutico devidamente capacitado para esta função. As análises bioquímicas de glicemia de jejum, do colesterol total (CT) e frações deste, HDL-C, LDL-C e VLDL-C foram realizadas em triplicata, considerando a média entre elas.

Para a determinação do risco cardiovascular foi calculada a pontuação para cada indivíduo a partir de parâmetros de sexo, idade, tabagismo, colesterol total e HDL-C, valor da pressão arterial sistólica não tratada e tratada, presença de DM e DA. A partir dos resultados foi estratificado o percentual de risco por meio dos Escores de Framingham (ERF) e pela Atualização da Diretriz Brasileira de Dislipidemias e Prevenção da Aterosclerose (2017) que sugere a Escala de Risco Global (ERG) após a classificação segundo a presença de DM e DA (Faludi et al., 2017).Após a atribuição da pontuação os jogadores foram estratificados em 3 categorias de acordo com o risco calculado. Para o ERF foram considerados como baixo risco os jogadores com probabilidade $<10 \%$, risco intermediário entre 10 e 20\% e alto risco > 20\% (Expert Panel on Detection, Evaluation, and Treatment of High Blood Cholesterol in Adults, 2001). Na avaliação pelo ERG foram considerados de baixo risco os jogadores com probabilidade $<5 \%$ de apresentarem os principais episódios cardiovasculares em 10 anos. No grupo de risco intermediário, foram considerados homens com $\geq 5 \%$ e $\leq 20 \%$ e mulheres com risco calculado $\geq 5 \%$ e $\leq 10 \%$. Foram considerados de alto risco aqueles com risco calculado $>20 \%$ para homens e $>$ $10 \%$ para mulheres (Faludi et al., 2017).

Os dados obtidos foram tabulados e analisados por meio do programa Statiscal Package for the Social Sciences (SPSS), versão 22.0 para Windows para os cálculos de frequência, média, desvio padrão e percentagens, bem como para construção dos gráficos e tabelas. 


\section{Resultados}

Participaram do estudo 13 jogadores, sendo 11 do sexo masculino e dois do sexo feminino, com idade entre 25 e 55 anos (38,2+10,1 anos). Destes, seis jogadores eram amputados, quatro eram lesados medulares, dois apresentavam sequelas do vírus da poliomielite e uma era usuário de prótese. Dentre os jogadores seis eram solteiros, quatro eram casados e três eram divorciados com predominância de ensino fundamental completo. Em relação ao tabagismo, encontraram-se dados bastante positivos, uma vez que apenas um jogador relatou ter este hábito (Tabela 1).

Tabela 1

Caracterização das varáveis sóciodemográficas dos jogadores de basquetebol em cadeira de rodas.

\begin{tabular}{|c|c|c|}
\hline & $\mathbf{n}$ & $\%$ \\
\hline \multicolumn{3}{|l|}{ Sexo } \\
\hline Masculino & 11 & $84,6 \%$ \\
\hline Feminino & 2 & $15,4 \%$ \\
\hline \multicolumn{3}{|l|}{ Idade } \\
\hline $20-29$ anos & 3 & $23,1 \%$ \\
\hline $30-39$ anos & 4 & $30,7 \%$ \\
\hline $40-49$ anos & 3 & $23,1 \%$ \\
\hline $50-59$ anos & 3 & $23,1 \%$ \\
\hline \multicolumn{3}{|l|}{ Deficiência } \\
\hline Amputação & 6 & $46,1 \%$ \\
\hline Lesão medular & 4 & $30,7 \%$ \\
\hline Sequela de Poliomietlite & 2 & $15,3 \%$ \\
\hline Uso de prótese & 1 & $7,69 \%$ \\
\hline \multicolumn{3}{|l|}{ Estado civil } \\
\hline Solteiro & 6 & $46,2 \%$ \\
\hline Casado & 4 & $30,7 \%$ \\
\hline Divorciado & 3 & $23,1 \%$ \\
\hline \multicolumn{3}{|l|}{ Escolaridade } \\
\hline Fundamental Incompleto & 3 & $23,1 \%$ \\
\hline Fundamental Completo & 4 & $30,7 \%$ \\
\hline Médio Incompleto & 2 & $15,4 \%$ \\
\hline Médio Completo & 2 & $15,4 \%$ \\
\hline Superior Completo & 2 & $15,4 \%$ \\
\hline \multicolumn{3}{|l|}{ Tabagismo } \\
\hline Sim & 1 & $7,7 \%$ \\
\hline Não & 12 & $92,3 \%$ \\
\hline
\end{tabular}

Fonte: Dados da pesquisa (2018).

Do total da amostra, somente um jogador relatou a presença de DM, sem uso de medicação específica e a maioria apresentou normoglicemia. Quanto à PA, nove jogadores apresentaram valores dentro da referência, três foram classificados como pré-hipertensos e um jogador foi classificado com hipertensão em estágio I. 
Na avaliação do perfil lipídico cinco jogadores apresentaram CT alto e oito estavam dentro do limite desejável. Para o LDL c foram identificados cinco jogadores acima do alvo terapêutico e oito dentro do alvo terapêutico. Já a avaliação do HDL c na amostra, identificou uma jogadora com valor adequado e uma com valor inferior aos parâmetros recomendados. Entre os homens três jogadores apresentaram valores inferiores e oito valores adequados à recomendação. Apenas dois jogadores foram classificados com valores de triglicérides alto (Tabela 2).

Tabela 2

Características clínicas dos jogadores de basquetebol em cadeira de rodas apresentadas por média e desvio padrão.

\begin{tabular}{|c|c|c|}
\hline Variáveis & Jogadores $(n=13)$ & Parâmetros \\
\hline Glicemia & $84,23 \pm 12,64$ & $<100 \mathrm{mg} / \mathrm{dl} *$ \\
\hline$P A S(m m H g)$ & $117,85 \pm 12,82$ & $<120 \mathrm{mmHg}^{* *}$ \\
\hline$P A D(m m H g)$ & $74,92 \pm 8,42$ & $<80 \mathrm{mmHg} * *$ \\
\hline Colesterol Total & $177,23 \pm 38,28$ & $<190 \mathrm{mg} / \mathrm{dl} * * *$ \\
\hline$L D L-C$ & $109,08 \pm 32,45$ & $\begin{array}{l}<130 \mathrm{mg} / \mathrm{dl}^{* * *} \text { - baixo } \\
<100 \mathrm{mg} / \mathrm{dl}^{* * *} \text { - intermediário } \\
<70 \mathrm{mg} / \mathrm{dl}^{* * *} \text { - alto } \\
<50 \mathrm{mg} / \mathrm{dl}^{* * *} \text { - muito alto }\end{array}$ \\
\hline$H D L-C$ & $\begin{array}{l}44,09 \pm 6,34(\mathrm{H}) \\
59,5 \pm 24,74(\mathrm{M})\end{array}$ & $\begin{array}{l}\geq 40 \mathrm{mg} / \mathrm{dl} * * *(\mathrm{H}) \\
\geq 50 \mathrm{mg} / \mathrm{dl} * * *(\mathrm{M})\end{array}$ \\
\hline Triglicérides & $114,15 \pm 50,84$ & $<150 \mathrm{mg} / \mathrm{d} 1^{* * *}$ \\
\hline
\end{tabular}

Legenda: PAS: Pressão Arterial Sistólica; PAD: Pressão Arterial Diastólica; LDL: Low Density Cholesterol; HDL: High Density Cholesterol; H: Homens; M: Mulheres

*SBD, $2017-2018$

**SBC, 2016

***DBDPA, 2017

Fonte: Dados da pesquisa (2018).

Quanto à avaliação antropométrica verificou-se que oito jogadores apresentaram classificação de IMC normal, quatro sobrepeso e um obesidade grau I. Na avaliação do risco de complicações metabólicas associadas à obesidade pela $\mathrm{CC}$, três não apresentaram risco, cinco risco aumentado e cinco risco muito aumentado. A avaliação do risco cardiovascular pelo ICO identificou cinco jogadores sem risco, três jogadores com baixo risco e cinco com alto risco. Já na avaliação do \%GC, dois jogadores foram classificados como abaixo da média, um dentro da média, quatro acima da média e seis com risco de doenças associadas à obesidade (Tabela 3).

Tabela 3

Dados antropométricos dos jogadores de basquetebol em cadeiras de rodas.

\begin{tabular}{lcc}
\hline & $\mathbf{n}$ & $\mathbf{\%}$ \\
\hline $\boldsymbol{I M C}^{*}$ & & \\
Nomal & 8 & 61,5 \\
Sobrepeso & 4 & 30,8 \\
Obesidade Grau I & 1 & 7,7
\end{tabular}




\begin{tabular}{lcc}
\hline & $\mathbf{n}$ & \% \\
\hline $\boldsymbol{C C}^{*}$ & 3 & \\
Sem risco & 5 & 23,1 \\
Risco aumentado & 5 & 38,5 \\
Risco muito aumentado & & 38,5 \\
ICO & & \\
Sem risco & 5 & 38,5 \\
Com baixo risco & 3 & 23,0 \\
Com alto risco & 5 & 38,5 \\
\% $\boldsymbol{G} \boldsymbol{C}^{*}$ & & \\
Abaixo da média & 2 & 15,4 \\
Dentro da média & 1 & 7,7 \\
Acima da média & 4 & 30,8 \\
Risco de doenças & 6 & 46,2 \\
\hline
\end{tabular}

Legenda: CC: circunferência da cintura; IMC: Índice de Massa

Corporal; ICO: Índice de Obesidade Central; GC: Gordura Corporal

*Rossi, 2017

Fonte: Dados da pesquisa, 2018.

Para predizer o risco para doenças cardiovasculares em um período de 10 anos para os jogadores de basquetebol, foram calculados os EF e ERG. A classificação pelo EF identificou, 12 jogadores com baixo risco de desenvolver DCV nos próximos 10 anos. Somente um jogador foi classificado com risco intermediário. Não foi encontrado nenhum jogador classificado com alto risco (Figura 1).

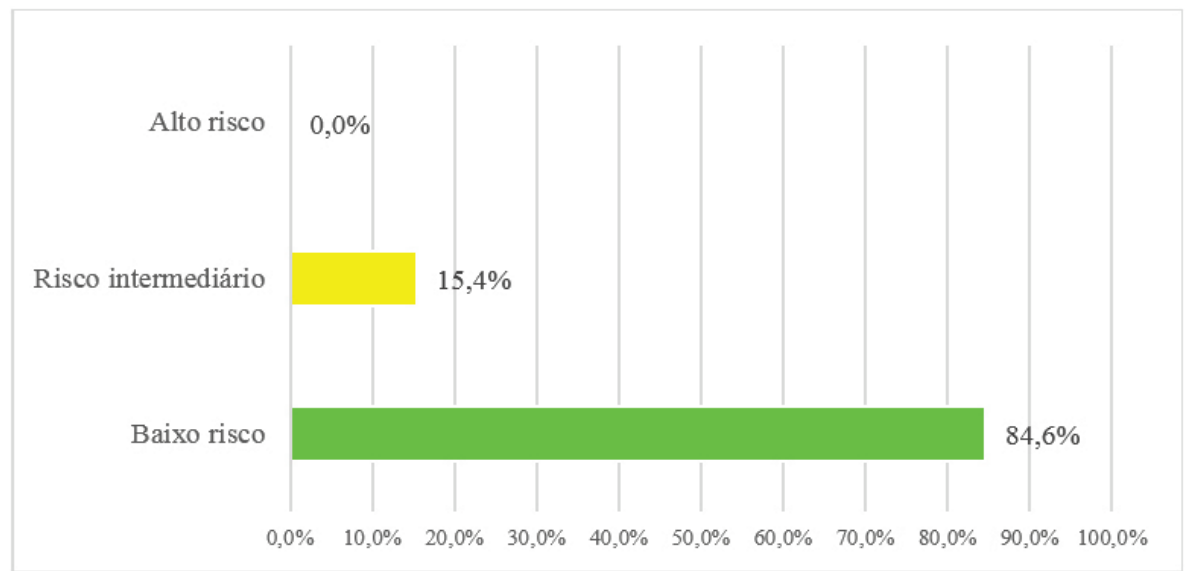

Figura 1. Distribuição dos jogadores de basquetebol em cadeiras de rodas quanto ao risco cardiovascular avaliado pelo Escore de Framingham. 
De acordo com o ERG sugerido pela DBDPA, dos jogadores avaliados, nove apresentaram baixo risco, dois risco intermediário, dois alto risco por DM e um muito alto risco por DA de desenvolver DCV nos 10 anos seguintes, conforme Figura 2.

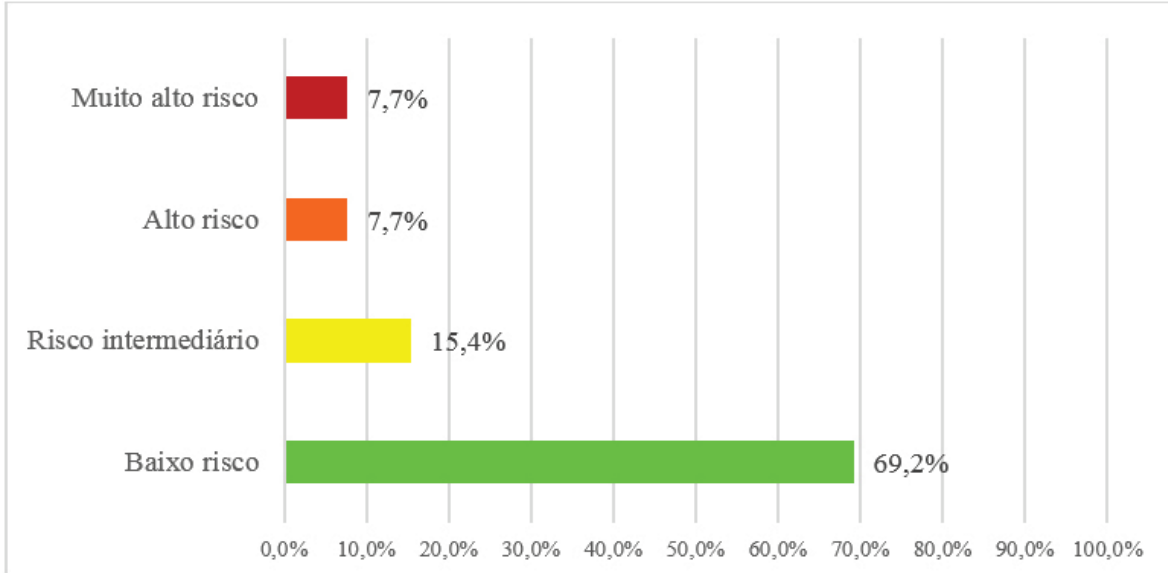

Figura 2. Distribuição dos jogadores de basquetebol em cadeiras de rodas quanto ao risco cardiovascular avaliado pela Escala de Risco Global.

\section{Discussão}

Foram encontrados poucos estudos sobre a avaliação dos fatores de risco cardiovasculares em pessoas com deficiência, particularmente em jogadores de basquetebol em cadeira de rodas. A maioria dos estudos investigou a presença desses fatores em indivíduos sem deficiência física (Candido et al., 2016; Oliveira, Ferreira \& Santos, 2016; Gama et al., 2017; Jahangiry et al., 2017; Cezar-Vaz et al., 2018). No entanto, o estudo realizado por Filho et al. (2006), avaliou atletas paraolímpicos com diferentes deficiências, sendo $14 \%$ lesados medulares. No presente estudo $46,1 \%$ dos jogadores eram amputados e 30,7 apresentavam lesão medular.

A faixa etária média encontrada foi de 38,2 anos, o que revela o predomínio de uma população adulta jovem. No entanto $46,2 \%$ dos pesquisados possuem idade acima de 40 anos, dado que não pode ser desconsiderado, uma vez que as DCV foram listadas entre as principais doenças crônicas não transmissíveis (DCNT) responsáveis por $51,6 \%$ do total de óbitos na população de 30 a 69 no Brasil no ano de 2015 (Ministério da Saúde, 2015). Isto reforça a importância de estimular a adoção ou manutenção de hábitos saudáveis para diminuição dos fatores de risco cardiovasculares na população estudada.

A maioria dos pesquisados $(84,6 \%)$ era do sexo masculino, assim como no estudo realizado por Filho et al. (2006), com atletas paraolímpicos, em que $71 \%$ da amostra era composta por homens. Ressalta-se que o basquetebol em cadeiras de rodas é uma modalidade praticada por atletas de ambos os sexos que tenham alguma deficiência físicomotora, segundo as regras adaptadas da Federação Internacional de Basquete em Cadeira de Rodas (International Wheelchair Basketball Federation [IWBF], 2017). 
Observou-se que grande parte dos jogadores eram escolarizados, com ensino fundamental completo, o que pode configurar um fator de proteção à saúde, uma vez que resultados do estudo de Candido et al., (2016) apontaram menor prevalência de fatores de risco comportamentais entre indivíduos com maior escolaridade.

Um dos fatores de risco pesquisados neste estudo foi o tabagismo, pela forte associação deste hábito com a ocorrência das DCV. Segundo a I Diretriz Brasileira de Prevenção Cardiovascular (2013), 50\% das mortes por DCV poderiam ser evitadas com a extinção do hábito de fumar (Simão et al., 2013). Nesse sentido, embora tenha sido identificado apenas um jogador tabagista, ressalta-se a importância da adoção de medidas que estimulem a diminuição ou cessação deste hábito por este jogador. Ademais torna-se necessário estimular a prevenção à iniciação ao hábito de fumar entre os outros participantes do estudo.

Quanto às características clínicas, os resultados da glicemia em jejum revelaram apenas um jogador com valor acima do recomendado para normoglicemia, pelas Diretrizes da Sociedade Brasileira de Diabetes 2017-2018 (Oliveira, Montenegro $\&$ Vencio, 2017). Ressalta-se que esse jogador não foi o que relatou a presença de DM, durante a aplicação do questionário. Os demais jogadores, inclusive aquele que relatou a presença de $\mathrm{DM}$, apresentaram valores dentro da recomendação. Isto revela um bom controle glicêmico no jogador diabético, mesmo sem o uso de medicação específica. O resultado dos valores médios de glicemia encontrados $(84,23 \pm 12,64)$ alinham-se aos resultados do estudo de Quintana e Neiva (2008) que revelou valores médios de normoglicemia $(87,48 \pm 6,48)$ em deficientes físicos praticantes de esportes adaptados. Nessa direção, o estudo de Phillips et al. (2004) evidenciou uma melhora na regulação da glicemia, decorrente de um programa de treinamento com suporte de peso corporal em esteira três vezes por semana durante seis meses em pessoas com lesão medular incompleta não praticantes de atividade física. Esse resultado evidência a importância da prática esportiva para o controle da glicemia sanguínea.

Embora os valores médios de PAS $(117,85 \pm 12,82)$ e PAD $(74,92 \pm 8,42)$ encontrados nos jogadores estejam em conformidade com a classificação normal da PA para adultos, segundo a $7^{\mathrm{a}}$ Diretriz Brasileira de Hipertensão Arterial (2016), foram identificados três jogadores com pré - hipertensão e um jogador com hipertensão em estágio I (Malachias et al., 2016). No jogador que havia relatado a presença de HAS, tratada com medicamento Enalapril os valores tanto da PAS quanto PAD estavam abaixo da classificação normal, segundo a mesma diretriz. Os valores de PA em conformidade na maioria dos jogadores, corroboram as evidências do estudo de Haddad, Silva, Barreto e Ferraretto (1997), no qual ocorreram diminuições significativas nos valores da PA em atletas deficientes após 12 semanas de um programa de treinamento supervisionado de membros superiores. Reitera-se a necessidade da adoção de medidas de intervenção específicas para controle da HAS, nos os jogadores identificados como pré-hipertensos e hipertensos.

Em relação ao perfil lipídico, apesar da média dos valores encontrados estarem dentro das recomendações, observou-se alguma alteração nos parâmetros analisados, CT, LDL-C, HDL-C e triglicerídeos. Os níveis de CT estavam elevados em 38,46\% dos jogadores assim como no estudo de Koyuncu, Yüzer, Yenigün e Özgirgin (2016), 
que encontrou colesterol total acima da meta terapêutica em 21,2\% dos 269 lesionados medulares avaliados. Esses resultados reforçam a importância da avaliação do CT em programas de rastreamento do risco cardiovascular, uma vez que o colesterol possui relação direta com as DCV, principalmente com valores acima de $200 \mathrm{mg} / \mathrm{dl}$, que acarretam riscos mais elevados (Faludi et al., 2017; Rodrigues \& Philippi, 2008).

Os valores do LDL-C dos jogadores foram classificados de acordo com a sugestão da Atualização da Diretriz Brasileira de Dislipidemias e Prevenção da Aterosclerose (2017) cujos pontos de corte variam de acordo com o risco cardiovascular estimado, sendo $130 \mathrm{mg} / \mathrm{dl}$ o valor de corte para baixo risco e para as demais categorias de maior risco os valores são menores (Faludi et al., 2017). A comparação dos resultados do LDL-C dos jogadores com essas recomendações, revelou elevado percentual de jogadores $(38,46 \%)$ com valores acima do alvo terapêutico. Percentuais mais elevados de indivíduos com LDL-C aumentado (52,9\%), considerando como ponto de corte $130 \mathrm{mg} / \mathrm{dl}$, foram encontrados no estudo de Pohl et al. (2018). Isso reforça a importância da avaliação desse parâmetro, uma vez que níveis elevados de LDL-C no plasma estão fortemente associados a DCV. Por outro lado, a redução das concentrações plasmáticas de LDL-C é evidenciada em muitos estudos como fator de prevenção do risco de desenvolvimento das DCV (Piepoli et al., 2016).

Os resultados médios do HDL-C revelaram valores acima da recomendação tanto para jogadores do sexo masculino $(44,09 \pm 6,34)$ quanto feminino $(59,5 \pm 24,74)$. O elevado percentual de jogadores com valores de HDL-C acima da recomendação $(69,23 \%)$ se assemelham aos resultados do estudo de Quintana e Neiva (2008), que identificou adequação do nível desse parâmetro em 60\% dos jogadores de basquetebol em cadeiras de rodas avaliados. Alguns estudos apontam que a prática de atividade física por deficientes físicos pode elevar os níveis de HDL-C colesterol (Brenes, Dearwater, Shapera, LaPorte \& Collins, 1986).

Embora neste estudo os resultados dos valores médios de triglicerídeos estejam abaixo da recomendação $(114,15 \pm 50,84)$, foi encontrado um pequeno percentual de jogadores $(15,38 \%)$ com esse parâmetro elevado. No entanto, percentuais mais elevados de triglicerídeos foram encontrados por Koyuncu et al. (2016), em seu estudo com indivíduos lesados medulares independente do nível de atividade física (31\% avaliados). Nessa direção, ressalta-se o papel dos triglicerídeos como fator de risco para as DCV, bem como a atenuação dos seus efeitos pela presença de elevados valores de HDL-C no plasma sanguíneo (Piepoli et al., 2016).

A avaliação da composição corporal dos jogadores identificou em todos os parâmetros pesquisados um elevado percentual de indivíduos com risco de DCV associadas à obesidade, mesmo com a prática do basquete em cadeiras de rodas. Embora a maioria dos jogadores tenham apresentado IMC adequado, se somados os jogadores classificados como sobrepeso e obesidade, esses representam aproximadamente $40 \%$ do total de avaliados. Elevadas taxas de sobrepeso e obesidade $(61,65 \%)$ também foram encontradas por Araújo et al. (2015) em seu estudo com um grupo de adolescentes com mielomeningocele praticantes de basquetebol em cadeira de rodas. Freire et al. (2017) em pesquisa acerca da produção científica sobre a prevenção das doenças cardiovasculares (DCV) no Brasil, encontrou em todos os 42 
artigos pesquisados, evidências da relação da obesidade, considerando IMC $>30$, como fator de risco para essas doenças. No entanto é importante salientar que a interpretação dos valores de IMC deve ser feita com cuidado, uma vez que o aumento do peso corporal pode não refletir uma condição de maior acúmulo de gordura corporal, mas uma consequência da participação de massa magra (Ribeiro, Melo \& Tirapegui, 2018).

Em virtude da limitação do IMC, foram aferidos também a CC, o ICO e o \% de GC dos jogadores de basquetebol em cadeiras de rodas com o objetivo de avaliar a gordura localizada na região abdominal além da distribuição da gordura corporal total. Na avaliação da CC, a maioria dos jogadores (77\%) apresentaram risco aumentado ou muito aumentado de complicações metabólicas associadas à obesidade. Esse dado corrobora os resultados de outros estudos que avaliaram a composição corporal de indivíduos com lesão de coluna vertebral e encontraram elevada concentração de gordura na região abdominal (Santos \& Guimarães, 2002; Donatto et al., 2008; Quintana \& Neiva, 2008; Eskici \& Ersoy, 2016). Esse acúmulo de gordura abdominal associado à inutilização dos membros inferiores dificultam o retorno venoso para o coração, sobrecarregando o órgão, levando à maior incidência de morte por doenças coronarianas em pacientes com lesões de coluna vertebral (Rodenbaugh, Collins, Nowacek, \& DiCarlo, 2003). Ademais, aproximadamente $40 \%$ dos jogadores apresentaram valores aumentados do ICO, o que predispõe essa população a risco metabólico e consequente maior risco cardiovascular.

Os resultados revelaram elevado $\% \mathrm{GC}(23,5 \% \pm 8)$ na maioria dos jogadores de basquetebol em cadeiras de rodas (77\%), indicando riscos de doenças associadas. Em pesquisa que utilizou método semelhante, Bulbulian et al. (1987), os autores Santos et al. (2016) também encontraram \% de GC acima da média $(26,54 \% \pm 7,9)$ em quase totalidade da amostra (91,66\%). De acordo com Keil, Zepetnek, Brooke-Wavell e Goosey-Tolfrey (2014), diversos estudos sugerem que mesmo entre atletas altamente treinados com deficiência são encontrados elevados percentuais de gordura corporal, indicando fator de risco para doenças cardiovasculares.

$\mathrm{O}$ risco de evento cardiovascular em dez anos, segundo o ERF, foi baixo para quase todos os jogadores $(84,6 \%)$, sendo que apenas um apresentou risco intermediário. Esse resultado pode ser atribuído à faixa etária entre 20 - 39 anos de mais da metade $(53,8 \%)$ da população estudada, além do elevado percentual $(69,2 \%)$ de jogadores com HDL-C acima da recomendação. O estudo de Filho et al. (2006) que também utilizou o ERF para avaliar o risco de evento cardiovascular em atletas paraolímpicos, encontrou resultados semelhantes em $70 \%$ da amostra. Os autores associaram o baixo risco cardiovascular à idade $(27,8 \pm 6,7)$ e ao treinamento físico dos atletas.

Neste estudo, apesar da maioria dos jogadores serem adultos jovens, a avaliação do risco de evento cardiovascular em dez anos a partir do ERG, identificou 15,4\% com risco intermediário, 7,7\% com alto risco e 7,7\% com muito alto risco. Ademais, o estudo de Gama et al. (2017) com jovens estudantes universitários, determinou risco intermediário segundo ERG em mais da metade $(52,38 \%)$ da amostra. Nesse sentido alguns estudos reforçam a importância dessa avaliação em populações jovens, que também estão sujeitas ao desencadeamento de alterações cardíacas pela associação de fatores de risco cardiovascular em longo prazo (Poletto, Schvartzman, Bruscato \& Moriguchi, 2018; Santos \& Ribas, 2017). 
A utilização de escores como prognóstico de eventos ou de mortalidade cardiovascular a partir de fatores de risco se justifica pela persistência no tempo e progressão das DCV (Tralhão et al., 2014). Principalmente quando utilizados para avaliar deficientes físicos praticantes de atividade física. Filho et al. (2006) afirmam que atletas paraolímpicos vivenciam fatores como estresse emocional e dificuldades financeiras, aliados à deficiência e sobrecarga cardiovascular causados pela dificuldade de locomoção, que podem ser prejudiciais à saúde.

\section{Conclusão}

Com base nos resultados deste estudo, pode-se concluir que nos jogadores de basquetebol em cadeiras de rodas há maior prevalência de sobrepeso e obesidade, elevados $\% \mathrm{GC}$ e CC, o que os predispõe a um risco acentuado para desenvolvimento de DCV. Ademais foram encontradas alterações nos perfis lipídicos e na PA, apesar de elevados valores de HDL-C.

Ainda, o uso da avaliação sugerida pela Atualização da Diretriz Brasileira de Dislipidemias e Prevenção da Aterosclerose identificou, em nosso estudo, jogadores com fatores de alto risco e muito alto risco para eventos cardiovasculares em dez anos, o que não foi evidenciado pela ERF. Com isso, a identificação dos fatores de riscos é útil para que ocorra uma maior prevenção das DCV e aconselhamento adequado sobre adoção de hábitos de vida saudáveis por esses indivíduos.

Por fim, ressalta-se a limitação metodológica do estudo, quanto à ausência de um instrumento de determinação de risco cardiovascular específico para a população estudada, fato este que requer certa atenção e urgência, visto que são cada vez mais crescentes fatores de risco cardiovasculares em deficientes físicos.

"O presente trabalho foi realizado com apoio da Coordenação de Aperfeiçoamento de Pessoal de Nível Superior - Brasil (CAPES) - Código do Financiamento 001"

"This study was financed in part by the Coordenação de Aperfeiçoamento de Pessoal de Nível Superior-Brasil (CAPES)- Finance code 001"

\section{Referências}

Araújo, P., Albornoz, S., Moura, H., Santos, S., Silva, R. C. da, \& Alvarenga M. (2015). Perfil da composição corporal de adolescentes praticantes de basquetebol de cadeira de rodas. Revista Brasileira de Nutrição Esportiva, 9(53), 498-505.

Brenes, G., Dearwater, S., Shapera, R., LaPorte, R. E., \& Collins, E. (1986) High density lipoprotein cholesterol concentrations in physically active and sedentary spinal cord injured patients. Archives of Physical Medicine and Rehabilitation, 67 (7), 445-50.

Bulbulian, E., Johnson, R. E., Gruber, J. J., \& Darabos, B. (1987). Body composition in paraplegic male athletes. Medicine \& Science in Sports \& Exercise, 19(3), 195-201.

Candido, R. L. R., Faria, C. C. C., Silva, J. P. F., \& Dantas, I. R. O. (2016). Avaliação de risco cardiovascular em colaboradores de um Centro Universitário. Revista Perquirere, 13(1), 158-172. 
Cezar-Vaz, M. R., Bonow, C.A., Mello, M. C. V.A. de, Xavier, D. M., Vaz, J. C., \& Schimith, M. D. (2018). Use of Global Risk Score for Cardiovascular Evaluation of Rural Workers in Southern Brazil. The ScientificWorld Journal. Article ID 3818065 (2018), 1-5. Donatto, F. F., Torres, C. A., Kano, F., Ferreira, D., Oliveira, M. R. M. de, \& Navarro, F. (2008). Comparação das medidas antropométricas entre atletas de basquete sobre cadeira de rodas e indivíduos fisicamente ativos. Revista Brasileira de Obesidade, Nutrição e Emagrecimento, 2(8), 201-205.

Eskici, G., \& Ersoy, G. (2016). An evaluation of wheelchair basketball players' nutritional status and nutritional knowledge levels. The Journal of Sports Medicine and Physical Fitness, 56(3), 259-68.

Expert Panel on Detection, Evaluation, and Treatment of High Blood Cholesterol in Adults (2001). Executive summary of the Third Report of the National Cholesterol Education Program (NCEP) Expert Panel on Detection, Evaluation, and Treatment of High Blood Cholesterol in Adults (Adult Treatment Panel III). Journal of the American Medical Association, 285(19), 2486-2497.

Faludi, A. A., Izar, M. C. O., Saraiva, JFK, Chacra, A. P. M., Bianco, H. T., Afiune Neto, A., Bertolami, A., Pereira, A. C., Lottenberg, A. M., Sposito, A. C..., \& Salgado Filho, W. (2017). Atualização da Diretriz Brasileira de Dislipidemias e Prevenção da Aterosclerose - 2017. Arquivos Brasileiros de Cardiologia, 109(1), 1-76.

Filho, J.A. O., Salvetti, X. M., Mello, M. T. de, Silva,A. C. da, \& Filho, B. L. (2006). Coronary risk in a cohort of paralympic athletes. British Journal of Sports Medicine, 40(11), 918-922.

Freire, A. K. da S., Alves, N. C. C., Santiago, E. J. P., Tavares, A. S., Teixeira, D. da S., Carvalho, I. A., Melo, M. C. P. de, \& Negro-Dellacqua, M. (2017). Panorama no brasil das doenças cardiovasculares dos últimos quatorze anos na perspectiva da promoção à saúde. Revista Saúde e Desenvolvimento, 11(9), 21-44.

Gama, W. M., Silva, D., Menezes, R. L. de, Garcia, B. M. S. P., Souza, L. C. A. e, Onofre, D. G., Silva, C. D, A. da, Pereira, G. L., Sanchez, F. F., Gonçalves, R. L., Marie, A. M. A., \& Leon, E. B. de (2017). Determinação do risco cardiovascular em adultos jovens universitários. Revista de Saúde Pública de Santa Catatarina, 10(3), 26-43.

Haddad, S., Silva, P. R. S., Barreto, A. C. P., \& Ferraretto, I. (1997). Efeito do treinamento físico de membros superiores aeróbio de curta duração no deficiente físico com hipertensão leve. Arquivos Brasileiros de Cardiologia, 69(3), 169-173.

International Wheelchair Basketball Federation (2017). Our Game. IWBF. Recuperado em: 15 de julho de 2019 de <https://iwbf.org/the-game/\#game-history>.

Jahangiry, L., Farhangi, M. A., \& Rezaei, F. (2017). Framingham risk score for estimation of 10-years of cardiovascular diseases risk in patients with metabolic syndrome. Journal of Health, Population and Nutrition, 36(1), 36-36.

Keil, M., Zepetnek, J. O. T. de, Brooke-Wavell, K., \& Goosey-Tolfrey, V. L. (2014). Measurement precision of body composition variables in elite wheelchair athletes, using dual-energy X-ray absorptiometry. European Journal of Sport Science, 16(1), 65-71.

Koyuncu, E., Yüzer, G. F. N., Yenigün, D., \& Özgirgin N. (2016). The analysis of serum lipid levels in patients with spinal cord injury. The Journal of Spinal Cord Medicine, 40(5) 567-572.

Malachias, M. V. B., Souza, W. K. S. B., Plavnik, F. L., Rodrigues, C. I. S., Brandão, A. A., Neves, M. F. T., Bortolotto, L. A., Franco, R. J. S., Poli-de-Figueiredo, C. E., 
Jardim, P. C. B. V., Amodeo, C..., \& Moreno, H. Júnior (2016). 7 a Diretriz Brasileira de Hipertensão Arterial. Arquivos Brasileiros de Cardiologia, 107(3), 1-83.

Mansur, A. P., \& Favarato, D. (2016). Tendências da Taxa de Mortalidade por Doenças Cardiovasculares no Brasil, 1980-2012. Arquivos Brasileiro de Cardiologia, [online], 107( 1), 20-25.

Ministério da Saúde (2015). Vigilância de Doenças Crônicas não Transmissíveis. Brasília: Ministério da Saúde. Recuperado em: 17 de maio de 2019 de $<\mathrm{http}$ ://portalms.saude. gov.br/saude-de-a-z/vigilancia-de-doencas-cronicas-nao-transmissiveis $>$.

Oliveira, A. C. M. de, Ferreira, R. C, \& Santos, A. A. (2016). Cardiovascular risk assessment according to the Framingham score and abdominal obesity in individuals seen by a clinical school of nutrition. Revista da Associação Médica Brasileira, 62(2), 138-144.

Oliveira, D. S., Tannus, L. R. M., Matheus, A. S. M., Corrêa, F. H., Cobas, R., Cunha, E. F. da, \& Gomes, M. B. (2007). Avaliação do Risco Cardiovascular Segundo os Critérios de Framingham em Pacientes Com Diabetes Tipo 2. Arquivos Brasileiros de Endocrinologia \& Metabologia, 51(2), 268-274.

Oliveira, J. E. P. de, Montenegro, R. M. Júnior, \& Vencio, S. (2017). Diretrizes da Sociedade Brasileira de Diabetes 2017-2018. São Paulo: Editora Clannad.

Organização das Nações Unidas (2015). Objetivos de Desenvolvimento Sustentável (ODS). Recuperado em 13 de julho de 2019: de <https://nacoesunidas.org/pos2015. ONU>.

Phillips S. M., Stewart, B. G., Mahoney, D. J., Hicks, A. L., McCartney, N., Tang, J. E., Wilkinson, S. B., Armstrong, D., \& Tarnopolsky, M. A. (2004). Bodyweight-support treadmill training improves blood glucose regulation in persons with incomplete spinal cord injury. Journal of Applied Physiology, 97(2), 716-724.

Piepoli, M. F., Hoes, H. W., Agewall, S., Albus, C.,Brotons, C., Catapano, A. L., Cooney, M. T., Corrà, U., Cosyns, B., Deaton, C., Graham, I..., \& Binno, S., ESC Scientific Document Group (2016). 2016 European Guidelines on cardiovascular disease prevention in clinical practice. European Heart Journal, 37(29), 2315-2381.

Platt, L. S. (2001). Medical and Orthopaedic Conditions in Special Olympics Athletes. Journal of Athletic Training, 36(1), 74-80.

Pohl, H. H., Arnold, E. F., Dummel, K. L., Cerentini, T. M., Reuter, E. M., \& Reckziegel, M. B. (2018). Indicadores antropométricos e fatores de risco cardiovascular em trabalhadores rurais. Revista Brasileira de Medicina do Esporte, 24(1), 64-68.

Poletto, S., Schvartzman, P. R., Bruscato, N. M., \& Moriguchi, E. (2018). Cohort study of cardiovascular risk in asymptomatic young adults: subclinical atherosclerosis and coronary calcium score. Anais da Academia Brasileira de Ciências, 90(3), 3129-3137

Quintana, R., \& Neiva, C. M. (2008). Fatores de risco para síndrome metabólica em cadeirantes: jogadores de basquetebol e não praticantes. Revista Brasileira de Medicina do Esporte, 14(3), 188-191.

Ribeiro, S. M. L., Melo, C. M. de., \& Tirapegui, J. (2018). Avaliação nutricional: teoria e prática (2a ed.). Rio de Janeiro : Guanabara Koogan.

Rodenbaugh, D. W., Collins, H. L., Nowacek, D. G., \& DiCarlo, S. E. (2003). Increased susceptibility to ventricular arrhythmias is associated with changes in $\mathrm{Ca} 2$ _regulatory proteins in paraplegic rats. American Journal of Physiology-Heart and Circulatory Physiology, 285(6), 2605-2613. 
Rodrigues, T. F. F., \& Philippi, S. T. (2008). Avaliação nutricional e risco cardiovascular em executivos submetidos a check-up. Revista da Associação Médica Brasileira, 54(4), 322-327.

Rossi, L. (2017). Avaliação nutricional: novas perspectivas (2a ed.). Rio de Janeiro: Guanabara Koogan.

Santos, D. M. G. dos, \& Ribas, S. A (2017). Dieta e fatores de risco para doença cardiovascular em escolares adolescentes: estudo transversal. Revista Adolescência e Saude, 15(2), 56-64.

Santos, M. V. L. dos, Batista, L. S., Tessarotto, V., Freitas, C. D., Fogaça, G. L. P. de A., \& Nacif, M. (2016). Perfil antropométrico e consumo alimentar de atletas de basquetebol em cadeira de rodas da região metropolitana de São Paulo. Revista Brasileira de Nutrição Esportiva, 10(58), 467-473.

Santos, S. S. dos, \& Guimarães F. J. de S. (2002). Avaliação antropométrica e de composição corporal de atletas paraolímpicos brasileiros. Revista Brasileira de Medicina do Esporte, 8(3), 84-91.

Simão, A.F., Précoma, D.B., Andrade, J.P., Correa, H. Filho, Saraiva, J.F.K., Oliveira, G.M.M., Murro, A.L.B., ... \& Souza W.K.S.B. (2013). I Diretriz Brasileira de Prevenção Cardiovascular. Sociedade Brasileira de Cardiologia. Arquivos Brasileiros de Cardiologia.: 101 (6Supl.2), 1-63.

Tralhão, A., Sousa, P. J., Ferreira, A. M., Miranda, M., Monge, J. C., Tomé, A., \& Duarte, J. M. (2014). Perfil de risco cardiovascular de adultos jovens saudáveis: evolução temporal. Revista Portuguesa de Cardiologia, 33(3), 147-154.

World Health Organization (2017). [homepage na Internet]. Cardiovacular Diseases (CVDs) 2017. WHO. Recuperado em: 17 de maio de 2019 de < http:/www.who.int/ mediacentre/factsheets/fs317/en/index.html>.

Recebido em: abril de 2019

Aceito em: abril de 2020

Kelen Cristina Estavanate de Castro: Nutricionista. Doutora em Promoção de Saúde pela Universidade de Franca (UNIFRAN). Docente do Centro Universitário de Patos de Minas (UNIPAM) nos cursos de Enfermagem, Medicina e Nutrição. Endereço: Rua Dolor Caixeta de Melo, 101. Bairro Aurélio Caixeta. Patos de Minas MG. CEP 38702080. Telefone: (034)988617286.

Ana Clara Garcia Guimarães: Bióloga e Nutricionista. Doutora em Ciência dos Alimentos pela Universidade Federal de Lavras (UFLA). Endereço: Rua Dr. Antônio Ferreira Maciel, 86 apto201. Bairro Várzea. Patos de Minas - MG. CEP: 38.700-363. Telefone: (34)992620333. Email: clara_gui14@yahoo.com.br

Guilherme Junio Silva: Discente do curso de Medicina do Centro Universitário de Patos de Minas (UNIPAM). Endereço: Av. Paranaíba, nº 2104. Bairro Brasil. Patos de Minas - MG. CEP 38700190. Telefone: (34)991817362. Email: guilherme.junio.silva@hotmail.com

Marconi Guarienti: Discente do curso de Medicina do Centro Universitário de Patos de Minas (UNIPAM). Endereço: Rua Diacuí, 99 apto. 401. Bairro: Caiçaras - Patos de Minas - MG. CEP: 38702-176. Telefone: (34)999074776. Email: marconi@unipam.edu.br

Maria Georgina Marques Tonello: Educadora Física. Doutora em Educação Especial pela Universidade Federal de São Carlos (UFSCar); Docente do Programa de Pós Graduação em Promoção de Saúde da Universidade de Franca (UNIFRAN). Endereço: Rua Siro Kaku, 72, Apt ${ }^{\circ}$ 71. Bairro Jardim Botânico. Ribeirão Preto - SP. CEP 14021614. Telefone: (016)997473232. Email: gina@ginatonello.com.br

Olímpio Pereira de Melo Neto: Farmacêutico. Mestre em Ciências Biológicas pela Universidade Federal de Ouro Preto (UFOP). Discente do curso de Medicina do Centro Universitário de Patos de Minas (UNIPAM). 
Endereço: Praça João Pinheiro, 17, apto 701, Centro, Patos de Minas - MG, CEP 38700-142. Telefone: (37)988232409. Email: opmeloneto@gmail.com

Karine Cristine de Almeida: Bióloga. Doutora em Imunologia e Parasitologia Aplicadas pela Universidade Federal de Uberlândia (UFU); Docente do Centro Universitário de Patos de Minas (UNIPAM). Endereço: Rua Rui Correa, 163. Apto 302. Bairro São Francisco. Patos de Minas-MG CEP38702018. Telefone: (34)988080931. Email: karineca@unipam.edu.br

Daniel dos Santos: Educador Físico. Doutor em Alimentos e Nutrição pela Universidade Estadual Paulista Júlio de Mesquita Filho (UNESP); Docente do Programa de Pós Graduação em Promoção de Saúde da Universidade de Franca (UNIFRAN). Endereço: Rua Barra $n^{\circ}$ 677. Bairro Jardim Continental - Patrocínio Paulista - SP. CEP14415-000 Telefone: (16)981116125. Email: daniel.santos@unifran.edu.br

E-mail para contato: kelen@unipam.edu.br 